\begin{abstract}
MS03 P15
Analysis of Carbohydrate Binding in a Family 5 Exoglucanase. John Cutfield, Yoshio Nakatani, Wayne Patrick, Sue Cutfield. Biochemistry Department, University of Otago, Dunedin, New Zealand.

E-mail: john.cutfield@stonebow.otago.ac.nz
\end{abstract}

Keywords: exoglucanase, site-directed mutagenesis, carbohydrate-binding

The exo-ß-1,3-glucanase from Candida albicans (Exg) functions in cell wall remodelling through its glucosyl hydrolase and transferase activities. As with other family 5 glycosyl hydrolases Exg possesses catalytic glutamate residues acting as nucleophile (E292) and proton donor (E192). The entrance to the active site pocket is flanked by a pair of antiparallel phenylalanine residues (F144 and F258). Crystallographic analysis of enzyme:inhibitor complexes has identified protein-sugar interactions at the 1 site and, to a lesser extent, at the $+1 /+2$ aromatic entranceway [1]. We have made various mutants to try and better identify sugar-binding sites, to alter the ratio of hydrolysis to transfer and to compare substrate specificity for hydrolase and glucosynthase reactions.

Mutations at the Phe-Phe gateway demonstrated that aromaticity must be preserved and that the transferase reaction was more sensitive to mutation, consistent with two docking events. The catalytically disabled mutant E292Q did not show coherent electron density to indicate stable binding of oligosaccharide substrates but unexpectedly revealed an external glucose binding site that may provide a foothold for the yeast cell wall Dglucan. Exg was converted to a glucosynthase via E292S and crystallized in the presence of donor 1-fluoro- $\alpha-\mathrm{D}$ glucose and acceptor p-nitrophenyl- $\beta$-D-glucopyranoside [2]. The two sugars were oriented such that donor sugar $\mathrm{C} 1$ was close to acceptor O6, consistent with $\mathrm{nmr}$ analysis of solution products which showed the main product to be ß-1,6-linked disaccharide and not $\beta-1,3$ - as expected.

[1] Cutfield SM, Davies GJ, Murshudov G, Anderson BF, Moody PC, Sullivan PA, Cutfield JF. J Mol Biol. 1999, 294, 771.

[2] Williams SJ, Withers SG, Carbohydr. Res. 2000, 327, 27.

\section{MS03 P16}

Crystallographic and kinetic studies of rhamnogalacturonan lyase from Aspergillus aculeatus

$\underline{\text { Sine Larsen }}^{\mathrm{a}, \mathrm{b}}$, Malene H. Jensen ${ }^{\mathrm{a}}$, Ulla Christensen ${ }^{\mathrm{a}}$, Leila Lo Leggio ${ }^{\mathrm{S}}$, Torben Borchert ${ }^{\mathrm{c}}$, Lars L. H. Christensen ${ }^{\mathrm{c}}$, ${ }^{a}$ Department of Chemistry, University of Copenhagen,Denmark,. $\quad{ }^{b}$ European Synchtrotron Radiaation Facility, Grenoble, France, ${ }^{c}$ Novozymes, Bagsvard,Denmark..E-mail: slarsen@esrf.eu

Keywords: enzymatic activity, polysaccharide lyase, catalytic residues

Rhamnogalacturonan lyase (AA-RGL) from A. aculeatus is a pectin backbone degrading enzyme that belongs to the polysaccharide lyase family 4 [1]. The structure of AARGL [2] was previously determined at a resolution of 1.5 $\AA$. The enzyme is comprised of three domains. The domains display structural homology with domains from other carbohydrate active enzymes, but neither of these domains possess any catalytic activity. Based on sequence alignments within polysaccharide lyase family 4 the location of the active site was proposed and possible candidates for the catalytic base and other active residues were identified [2]. Two of these putative active site residues were mutated (K150A and H210A). Preliminary kinetic studies have shown that the two mutants of AARGL have low catalytic activity. The two mutant enzymes could be crystallized in conditions similar to those of the native enzyme, 20\% PEG 4000, $0.1 \mathrm{M}\left(\mathrm{NH}_{4}\right)_{2} \mathrm{SO}_{4}$ and 0.1 $\mathrm{M}$ sodium acetate $\mathrm{pH}$ 4.3. Beautiful single plate-like crystals of both mutants were obtained by seeding from plate clusters of the H210A mutant. The crystals of both mutants diffract to a resolution better than $1.9 \AA$ on a in house equipment. The diffraction data revealed a tetragonal space group, $\mathrm{P} 4_{3} 2_{1} 2$, like the native enzyme. Cell dimensions were $\mathrm{a}=\mathrm{b}=77.0 \AA$ and $\mathrm{c}=170 \AA$. Preliminary structure determination show that the mutations do not disturb the overall structure, consistent with a direct role of $\mathrm{K} 150$ and $\mathrm{H} 210$ in catalysis or substrate binding.

[1]. Coutinho PM \& Henrissat B. (1999) Carbohydrate-Active Enzymes server at URL: http://afmb.cnrs-mrs.fr/CAZY/

[2]. McDonough MA, Kadirvelraj R, Harris P, Poulsen JC, Larsen S. (2004), FEBS Lett. 565, 188-94

\section{MS03 P17}

Structure of the full-length response regulator PleD in its activated state Tilman Schirmer,Paul Wassmann, Dietrich Samoray, Biozentrum, University of Basel, Switzerland. E-mail: tilman.schirmer@unibas.ch

Keywords: allosteric enzymes, signal transduction, response regulator

During the last decade cyclic di-GMP has become recognized as a novel ubiquitous second messenger in eubacteria [1]. Condensation of two GTP to the dinucleotide is catalyzed by two identical diguanylate cyclase domains (DGC or GGDEF) occurring in various combinations with sensory and regulatory modules. One of these modular proteins is $\mathrm{PleD}-\mathrm{a}$ response regulator receiver composed of two CheY like domains and a DGC domain [2], thus merging "two-component" signal transduction with c-diGMP signaling. PleD is involved in the cell morphology development of the marine bacteria Caulobacter crescentus, namely conversion of the motile (flagelled) to the sessile (stalked) form [3].

Like other response regulators, PleD is activated by a cognate histidine kinase through phosphorylation at Asp53 of the first CheY-like domain. Since phospho-aspartates are short-lived, structure analysis of the activated state is challenging. The problem was overcome by chemical modification with $\mathrm{BeF}_{3}^{-}$, which was previously shown for CheY to act as a phosphate analog ${ }^{4}$. Such activated PleD was crystallized in a new crystal form showing the modified Asp affecting adjacent residues e.g. Thr85, Phe102 and causing large changes in the $\beta 4-\alpha 4$ loop as seen before for response regulator receivers [4]. These movements result in a relative rearrangement of the CheYlike domains of a PleD monomer, which allows the formation of a tight dimer, bringing the two DGC domains in near proximity to each other. Such a dimer is the basis for the catalytic activity of PleD, namely formation of cdiGMP.

PleD shows non-competitive product inhibition. An allosteric c-diGMP binding site between the DGC-domain and the second CheY-like domain had been identified 
before [2]. Here, another c-diGMP binding mode is observed that cross-links the two DGC domains of the dimer. This new mode of domain immobilization may be of general importance for a large sub-class of DGC proteins.

[1] Jenal, U.; Malone. J. Annu. Rev. Genet. 40:385-407 (2006). [2] Chan, C.; Paul, R.; Samoray, D.; Amiot, N.C.; Giese,B.; Jenal,U.; Schirmer, T. Proc. Natl. Acad. Sci. USA 101, 17084 (2004).

[3] Aldridge, P.; Paul, R.; Goymer, P.; Rainey, P.; Jenal, U. Mol. Microbiol. 47:1695-708 (2003).

[4] Lee, S.Y.; Cho, H.S.; Pelton, J.G.; Yan, D.; Berry, E.A.; Wemmer, D.E. J. Biol. Chem. 276:16425-31 (2001).

\section{MS03 P18}

Structural characterization of threonine aldolases

Michael Uhl ${ }^{\mathrm{a}}$, Kerstin Prettler ${ }^{\mathrm{a}}$, Christoph Reisinger ${ }^{\mathrm{a}}$, Karl

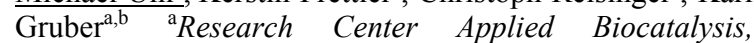
Petersgasse 14, A-8010 Graz, Austria, ${ }^{b}$ Department of Chemistry, University of Graz, Heinrichstr. 28, A-8010 Graz, Austria. E-mail: michael.uhl@a-b.at

Threonine aldolases (TAs) are PLP dependent enzymes which catalyze the reversible conversion of $\beta$-hydroxy- $\alpha$ aminoacids into glycine and the corresponding aldehyde. In general it's possible to distinguish between two main types, L-selective and D-selective threonine aldolases, depending on their enantioselectivity with respect of the $\alpha$-carbon of the amino acid. Threonine aldolases possess a high potential as biocatalysts for the synthesis of $\beta$ hydroxy- $\alpha$-amino acids which are important chiral pharmaceutical intermediates.

We are specifically working with two bacterial enzymes, an LTA and a DTA, which share only very low sequence similarity.

For LTA, a microbatch screen was performed, using solutions of the Index Screen (Hampton Research) which yielded first conditions. After optimization it was possible to collect a dataset to a maximum resolution of $3.5 \AA$ at our home x-ray source. The thus obtained LTA crystals are monoclinic (spacegroup $\mathrm{P} 2_{1}$ ) with unit cell dimensions $\mathrm{a}=106.5 \AA ; \mathrm{b}=116.1 \AA ; \mathrm{c}=106.8 \AA$ and $\beta=93.0^{\circ}$. The asymmetric unit consists of one homotetramer of the enzyme and contains about $70 \%$ solvent. First steps towards a structure solution by molecular replacement have been performed using this dataset and a previously generated homology model of LTA.

For DTA we had to employ reductive methylation of lysine residues (using formaldehyde and $\mathrm{NaBH}_{4}$ ) in order to obtain diffraction quality crystals. With the thus modified DTA a standard crystallization screen was carried out. At our home x-ray source, first crystals yielded a diffraction pattern up to a maximum resolution in the range of $3.5 \AA$. They belong to spacegroup $\mathrm{P} 2{ }_{1}$ with unit cell dimensions of $\mathrm{a}=62.4 \AA ; \mathrm{b}=83.8 \AA$; $=73.3 \AA$ and $\beta=112.1^{\circ}$.

Further optimization of the crystallization conditions in order to improve the resolution of the diffraction data, as well as soaking experiments with substrates and inhibitors are underway.

\footnotetext{
MS03 P19

Crystallographic studies of an active-site mutant of plant-type L-asparaginase Karolina Michalska ${ }^{1}$, Alejandra Hernandez-Santoyo ${ }^{1}$, Mariusz Jaskolski ${ }^{1,2}{ }^{1}$ Dept of Crystallography, Faculty of Chemistry, A.Mickiewicz Univ., Poznan, Poland; ${ }^{2}$ Center for Biocrystallographic
}

Research, Inst. of Bioorg. Chem., Pol. Acad. Sci., Poznan, Poland. E-mail: dziuba@amu.edu.pl

Keywords: plant-type asparaginase, isoaspartyl
peptidase, Ntn-hydrolase, autoactivation

Plant asparaginases hydrolyze the side-chain amide bond of asparagine or of isoaspartyl dipeptides. Close homologs have been found not only in plants, but also in bacteria, with the E. coli protein, EcAIII, being particularly well studied. The enzymes from both sources belong to the family of Ntn-hydrolases with a catalytic Thr nucleophile located at the free $\mathrm{N}$-terminus of subunit $\beta$, liberated during an autoproteolytic maturation event which generates two separate subunits $(\alpha$ and $\beta$ ) from a singlechain precursor. It is believed that the maturation mechanism involves the very same Thr nucleophile that is liberated in its consequence. However, the details of the maturation reaction are obscure, as the free amino group, supposed to activate the nucleophilic hydroxyl, is not present in the immature protein. To investigate the structural basis of EcAIII maturation, we have generated and crystallized an active-site mutant with the catalytic Thr residue substituted by alanine (T179A). The structure has been solved in two crystal forms, one of which corresponds to that of the wild-type enzyme. The protein exists as a dimer resembling the $(\alpha \beta)_{2}$ oligomeric form of the mature enzyme. The linker connecting the $\alpha$ and $\beta$ portions of the precursor can be traced nearly in its entirety, allowing a discussion of a nucleophilic attack launched by residue 179 on the preceding peptide bond. In variance with the wild-type structure, a fragment of the linker chain is seen in the active-site cavity. A tightly coordinated sodium cation, which in the mature protein shapes a loop supporting the active site elements, is found in an analogous structure of the T179A mutant. This suggests that the metal cation is coordinated at an early stage during the protein folding process and that its presence is essential for both the maturation reaction and the final L-asparaginase activity.

\section{MS03 P20 \\ Structural Properties and Electrostatics of Cold-active beta-Galactosidase Tereza Skálováa, Jan Dohnálek ${ }^{\mathrm{a}}$, Andrea Štěpánkováa, Petra Lipovováb, Vojtěch Spiwok ${ }^{\mathrm{b}}$, Jarmila Duškováa $^{a}$, Petr Kolenko ${ }^{a}$, Hana Petrokováa ${ }^{a}$, Hynek Strnad $^{\mathrm{b}}$, Blanka Králováb, Jindřich Hašek ${ }^{\mathrm{a}}{ }^{a}$ Institute of Macromolecular Chemistry ASCR, v.v.i, Heyrovského nám. 2, 16206 Praha 6, Czech Republic. ${ }^{b}$ Institute of Chemical Technology, Technická 5, 16628 Praha 6, Czech Republic. \\ E-mail: skalova@imc.cas.cz}

\section{Keywords: beta-galactosidase, cold-active, hexamer}

Arthrobacter sp. C2-2, a soil bacteria found on an island near Antarctica, belongs to psychrotrophic, i.e. cold tolerant, microorganisms. Structure of its betagalactosidase (hydrolase cleaving lactose into galactose and glucose), iso-enzyme C-2-2-1, was solved up to $1.9 \AA$ resolution.

The beta-galactosidase belongs to glycosyl hydrolase structural family 2 and has $30 \%$ sequence identity with Escherichia coli beta-galactosidase. In spite of the chain similarity, both enzymes differ in their oligomerization states. 